\title{
APPLICATIONS OF INVARIANT TRANSFORMATIONS IN ONE-DIMENSIONAL NON-STEADY GASDYNAMICS*
}

BY

\author{
S. P. CASTELL (Bearing Research Centre, RHP Ltd. Chelmsford)** \\ $\Lambda \mathrm{ND}$ \\ C. ROGERS, (Old Dominion University, Norfolk, Virginia)***
}

Summary. Certain reciprocal and adjoint transformations available for onedimensional non-stcady gasdynamic flow are applicd to an existing solution to construct new exact solutions of the governing equations. The particle trajectories and the pressure-density relations on these trajectories are calculated. An application of the adjoint transformation in studying the flow between a piston and a non-uniform shock wave is indicated.

1. Introduction. The development and application of transformation theory in gasdynamics and magnetogasdynamics has a long history. In particular, the subject of the invariance properties of the governing equations has received considerable attention. The work of Bateman [1], Haar [2] and Prim [3] established the invariance of the gasdynamic equations under certain important transformations. These were later discussed by Tsien [4], Bateman [5] and Power and Smith [6] and further developments were made more recently by Power and Tunbridge [7], Power and Walker [8], Nikol'skii [9], Smith [10], Tomilov [11], Rykov [12], Ustinov [13] and Movsesian [14] when results were derived for both unsteady and magnetogasdynamic flows.

Some applications of an invariant transformation in one-dimensional non-steady gasdynamics were given by Ustinov [13] and in a later paper an example of a generalization of this transformation and its use was given. The governing equations of onedimensional non-steady flow were taken in the form

$$
\begin{aligned}
\rho_{t}+(\rho u)_{x} & =0, \\
\rho\left(u_{t}+u u_{x}\right)+p_{x} & =0, \\
s(p, \rho) & =F(\tau),
\end{aligned}
$$

where $p, \rho, u, s$ are respectively pressure, density, speed and entropy, and $F$ is an arbitrary function; and Ustinov found that under the transformation

$$
\begin{aligned}
d \tau & =\rho d x-\rho u d t, \\
d \xi & =\rho u d x-\left(p+\rho u^{2}\right) d t,
\end{aligned}
$$

the governing equations were invariant provided new flow variables $P, R, U$, $S$ (each

* Received February 22, 1972; revised version received January 4, 1973.

** Now with Touche Ross \& Co., 27 Chancery Lane, London WC2A INF.

*** Now at University of Western Ontario, Canada. 
functions of $\tau, \xi$ ) were introduced according to

$$
p=R^{-1}, \quad \rho=P^{-1}, \quad u=U, \quad S=s
$$

and

$$
d x=\left(P+R U^{2}\right) d \tau-R U d \xi, \quad d t=R U d \tau-R d \xi .
$$

Here $\tau$ is the usual stream-function and $\xi$ that first introduced by Martin [15].

This transformation was then used, in particular, to generate a new solution for non-steady one-dimensional homentropic perfect gas flow from a solution derived by Sedov [16]. Ustinov also discussed an application of the transformation in an analysis of the flow between a piston and a non-uniform shock moving into a gas at rest.

Here we follow the work of Ustinov and, in a similar manner, apply the reciprocal and adjoint transformations given by Rogers $[17,18]$ to the Sedov solution. It is to be noted that under the Ustivov transformation (1.1)-(1.7), for homentropic flow, the perfect gas law of the original flow maps into a perfect gas law for the transformed flow. However, under reciprocal and adjoint transformations a given perfect gas solution is associated with a multi-parameter family of solutions in the reciprocal and adjoint spaces, each member having its own equation of state.

2. The Sedov solution under the reciprocal transformation. The exact homentropic solution of Eqs. (1.1)-(1.3) which Sedov [16] found for a perfect gas depends on an arbitrary function of the time $t$ which takes different forms according to the value of the adiabatic exponent $\gamma$. The solution is (Ustinov [13])

$$
\begin{aligned}
& u(x, t)=(1-\epsilon) \frac{x_{0}}{t_{0}} r\left(1+\sigma^{-2 \epsilon}\right)^{1 / 2}, \\
& \rho(x, t)=\rho_{0}\left[\frac{\epsilon^{2} x_{0}^{2} \rho_{0}}{\gamma p_{0} t_{0}{ }^{2}}\left(1+r^{2}\right) \sigma^{-2 \epsilon}\right]^{(1-\epsilon) / 2 \epsilon}, \\
& p(x, t)=\frac{p_{0}}{\rho_{0}{ }^{\gamma}} \rho^{\gamma}=\frac{p_{0}}{\rho_{0}{ }^{\gamma-1}}\left[\frac{\epsilon^{2} x_{0}^{2}}{\gamma p_{0} \rho_{0} t_{0}^{2}}\left(1+r^{2}\right) \sigma^{-2 \epsilon}\right]^{(1+\epsilon) / 2 \epsilon},
\end{aligned}
$$

where $\epsilon=(\gamma-1) /(\gamma+1), \gamma>1, x_{0}, t_{0}, p_{0}, \rho_{0}$ are arbitrary constants,

$$
r(x, t)=\frac{x}{x_{0}} \sigma^{\epsilon-1},
$$

and where $\sigma(t)$ is a function of the time $t$, arbitrary to the extent that it depends on the value of $\epsilon$, and defined by

$$
d t=t_{0}\left(1+\sigma^{2 \epsilon}\right)^{-1 / 2} d \sigma .
$$

This solution was also discussed by Nikol'skii [9] and Ustinov followed him in presenting it in the above form.

Now Rogers [17] found that under the transformation

$$
\begin{gathered}
x^{\prime}=x . \\
a_{1} d t^{\prime}=\rho u d x-\left(p+\rho u^{2}+a_{2}\right) d t
\end{gathered}
$$


the equations (1.1)-(1.3) were invariant provided that new reciprocal flow variables $p^{\prime}, \rho^{\prime}, u^{\prime}, s^{\prime}$ (each functions of $x^{\prime}, t^{\prime}$ ) were introduced according to

$$
\begin{aligned}
& p^{\prime}=a_{4}-a_{1}{ }^{2} a_{3} /\left(p+a_{2}\right), \\
& \rho^{\prime}=a_{3}\left(p+a_{2}\right) \rho /\left(p+\rho u^{2}+a_{2}\right), \\
& u^{\prime}=-a_{1} u /\left(p+a_{2}\right), \\
& s^{\prime}=s^{\prime}(s),
\end{aligned}
$$

and the $a_{i}(i=1,2,3,4)$ are arbitrary (constant) parameters.

Thus, given the homentropic Sedov solution (2.1)-(2.5) $p, \rho, u, s$ (each functions of $x, t$ ) of Eqs. (1.1)-(1.3), a four-parameter family of solutions may be constructed in $\left(x^{\prime}, t^{\prime}\right)$-space, given by $(2.8)-(2.11)$, together with $s^{\prime}=$ constant and

$$
x^{\prime}=x, \quad a_{1} d t^{\prime}=f(x, \sigma) d x+g(x, \sigma) d \sigma,
$$

where

$$
\begin{aligned}
& f(x, \sigma)=\frac{(1-\epsilon) \rho_{0} x_{0}}{t_{0}}\left[\alpha\left(1+\frac{x^{2}}{x_{0}} \sigma^{2 \epsilon-2}\right) \sigma^{-2 \epsilon}\right]^{(1-\epsilon) / 2 \epsilon} \frac{x}{x_{0}} \sigma^{\epsilon-1}\left(1+\sigma^{-2 \epsilon}\right)^{1 / 2}, \\
& g(x, \sigma)=-\left\{\frac{p_{0}}{\rho_{0}^{\gamma-1}}\left[\alpha\left(1+\frac{x^{2}}{x_{0}^{2}} \sigma^{2 \epsilon-2}\right) \sigma^{-2 \epsilon}\right]^{(1+\epsilon) / 2 \epsilon}\right. \\
& \left.+\frac{(1-\epsilon)^{2} \rho_{0} x_{0}{ }^{2}}{t_{0}{ }^{2}}\left[\alpha\left(1+\frac{x^{2}}{x_{0}{ }^{2}} \sigma^{2 \epsilon-2}\right) \sigma^{-2 \epsilon}\right]^{(1-\epsilon) / 2 \epsilon} \frac{x^{2}}{x_{0}{ }^{2}} \sigma^{2 \epsilon-2}\left(1+\sigma^{-2 \epsilon}\right)+a_{2}\right\} t_{0}\left(1+\sigma^{2 \epsilon}\right)^{-1 / 2} \text {, } \\
& \alpha=\epsilon^{2} x_{0}^{2} \rho_{0} / \gamma p_{0} t_{0}^{2} \text {. }
\end{aligned}
$$

It is readily verified that

$$
\int f(x, \sigma) d x=\frac{\epsilon(1-\epsilon) \rho_{0} x_{0}^{2}}{(1+\epsilon) t_{0}} \alpha^{(1-\epsilon) / 2 \epsilon}\left(1+\sigma^{-2 \epsilon}\right)^{1 / 2}\left(1+\frac{x^{2}}{x_{0}^{2}} \sigma^{2 \epsilon-2}\right)^{(1+\epsilon) / 2 \epsilon}
$$

and setting

$$
\begin{aligned}
\frac{p_{0} \alpha^{(1+\epsilon) / 2 \epsilon} t_{0}}{\rho_{0}^{\gamma-1}} & =1, \\
\frac{(1-\epsilon)^{2} \rho_{0} \alpha^{(1-\epsilon / 2 \epsilon}}{t_{0}} & =1, \\
a_{2} t_{0} & =1, \\
\frac{\epsilon(1-\epsilon) \rho_{0} x_{0}{ }^{2} \alpha^{(1-\epsilon) / 2 \epsilon}}{(1+\epsilon) t_{0}} & =a_{1}, \\
x_{0} & =1,
\end{aligned}
$$

then we get

$$
a_{1} t^{\prime}=a_{1}\left(1+\sigma^{-2 \epsilon}\right)^{1 / 2}\left(1+x^{2} \sigma^{2 \epsilon-2}\right)^{(1+\epsilon) / 2 \epsilon}-\int g_{1}(x, \sigma) d \sigma-a_{1}
$$

where 


$$
\begin{aligned}
g_{1}(x, \sigma)= & {\left[\left(1+x^{2} \sigma^{2 \epsilon-2}\right) \sigma^{-2 \epsilon}\right]^{(1+\epsilon) / 2 \epsilon}\left(1+\sigma^{2 \epsilon}\right)^{-1 / 2} } \\
& +x^{2}\left[\left(1+x^{2} \sigma^{2 \epsilon-2}\right) \sigma^{-2 \epsilon}\right]^{(1-\epsilon / 2 \epsilon)} \sigma^{2 \epsilon-2}\left(1+\sigma^{-2 \epsilon}\right)\left(1+\sigma^{2 \epsilon}\right)^{-1 / 2}+\left(1+\sigma^{2 \epsilon}\right)^{-1 / 2} .
\end{aligned}
$$

Eqs. (2.13) and (2.15) give

$$
a_{1}=\epsilon /\left(1-\epsilon^{2}\right)=\left(\gamma^{2}-1\right) / 4 \gamma .
$$

The equation to the particle trajectories is given by $d x^{\prime}=u^{\prime} d t^{\prime}$; in view of (2.6), (2.7) and (2.10) this yields simply

$$
d x=u d t .
$$

Thus, under this reciprocal transformation particle trajectories transform to particle trajectories. This is not to say that the particle trajectories will have the same shape in both spaces; in view of (2.5), (2.17) may be written

$$
d x=\frac{(1-\epsilon) x_{0}}{t_{0}} \frac{x}{x_{0}} \sigma^{\epsilon-1}\left(1+\sigma^{-2 \epsilon}\right)^{1 / 2} t_{0}\left(1+\sigma^{2 \epsilon}\right)^{-1 / 2} d \sigma .
$$

Since $x^{\prime}=x$, this integrates to give

$$
x^{\prime}=A \sigma^{(1-\epsilon)},
$$

$A$ an arbitrary constant. The equation to the particle trajectories in the reciprocal space is now given parametrically by (2.18) and

$$
\begin{aligned}
a_{1} t^{\prime}=a_{1}\left(1+\sigma^{-2 \epsilon}\right)^{1 / 2}\left(1+A^{2}\right)^{(1+\epsilon) / 2 \epsilon} & \\
& -\left(1+A^{2}\right)^{(1+\epsilon) / 2 \epsilon} I_{1}-A^{2}\left(1+A^{2}\right)^{(1-\epsilon) / 2 \epsilon} I_{2}+I_{3}-a_{1},
\end{aligned}
$$

where

$$
\begin{aligned}
& I_{1}=\int \sigma^{-(1+\epsilon)}\left(1+\sigma^{2 \epsilon}\right)^{-1 / 2} d \sigma, \\
& I_{2}=\int \sigma^{-(1+\epsilon)}\left(1+\sigma^{2 \epsilon}\right)^{1 / 2} d \sigma, \\
& I_{3}=\int\left(1+\sigma^{2 \epsilon}\right)^{-1 / 2} d \sigma .
\end{aligned}
$$

Introducing a new parameter $\theta$ by $\sinh ^{2} \theta=\sigma^{2 \epsilon}$, we have the particle trajectorics given parametrically by

$$
\begin{gathered}
x^{\prime}=A \sinh ^{(1-\epsilon) / \epsilon} \theta, \\
t^{\prime}=\frac{1}{a_{1}}\left\{\epsilon^{-1}\left(1+A^{2}\right)^{(1-\epsilon) / 2 \epsilon}\left[\left(a_{1} \epsilon+2\right) A^{2}+\left(1+a_{1} \epsilon\right)\right] \operatorname{coth} \theta\right. \\
\left.-\epsilon^{-1} A^{2}\left(1+A^{2}\right)^{(1-\epsilon) / 2 \epsilon} \theta+I_{3}(\epsilon, \theta)-a_{1}\right\},
\end{gathered}
$$

with $a_{1}=\epsilon\left(1-\epsilon^{2}\right)^{-1}, \epsilon=\frac{1}{2}, \frac{1}{3}, \frac{1}{5} ; I_{3}\left(\frac{1}{2}, \theta\right)=2 \cosh \theta, I_{3}\left(\frac{1}{3}, \theta\right)=\frac{3}{2}\left(\frac{1}{2} \sinh 2 \theta-\theta\right)$, and $I_{3}\left(\frac{1}{5}, \theta\right)=5 / 4\left(\frac{1}{8} \sinh 4 \theta-\sinh 2 \theta+\frac{3}{2} \theta\right)$.

Fig. 1 shows a typical particle trajectory, given by $A=1$, in the three cases $\epsilon=$ $\frac{1}{2}, \frac{1}{3}, \frac{1}{5}$. 


$$
\text { t' }
$$

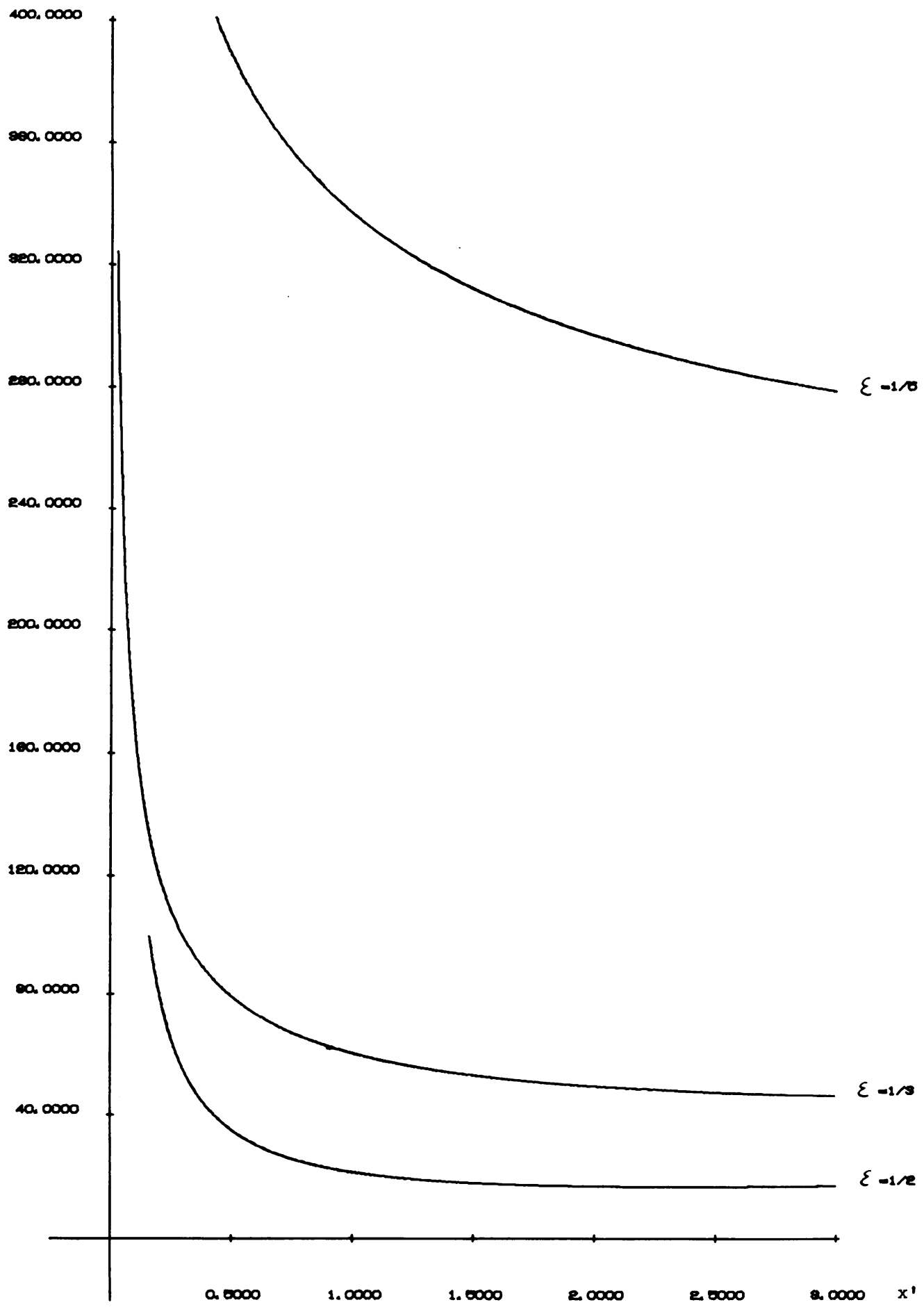

FIG. 1. Reciprocal transformation: particle trajectory $A=1$ for the three cases $\xi=\frac{1}{2}, \frac{1}{3}, \frac{1}{5}$. 
From (2.1)-(2.4) and in view of (2.12)-(2.16) we have

$$
\begin{aligned}
& p=\frac{\left(1+A^{2}\right)^{(1+\epsilon) / 2 \epsilon}}{t_{0}} \sigma^{-(1+\epsilon)}, \\
& \rho=\frac{t_{0}\left(1+A^{2}\right)^{(1-\epsilon) / 2 \epsilon}}{(1-\epsilon)^{2}} \sigma^{-(1-\epsilon)}, \\
& u=\frac{(1-\epsilon)}{t_{0}}\left(1+\sigma^{-2 \epsilon}\right)^{1 / 2}
\end{aligned}
$$

along particle trajectories $x=x^{\prime}=A \sigma^{(1-\epsilon)}$ in the reciprocal space. Substituting these values in (2.8) and (2.9) yields $p^{\prime}$ and $\rho^{\prime}$ as functions of $\sigma$ along the particle trajectories. Fig. 2 gives the result of this substitution, showing $p^{\prime}$ plotted against $\rho^{\prime}$ for the case $A=0, \epsilon=\frac{1}{5}, a_{1}=0.208$ and $a_{2}=a_{3}=a_{4}=1$. Also shown is the curve $\rho^{\prime \gamma}$, that is ${\rho^{\prime 3 / 2}}^{\prime 3}$ and it is seen that the gencral shapes of the curves agree well. This indicates that, by suitable choice of values for $a_{i}(i=2,3,4)$, the flow in the reciprocal space could be made to approximate closely that of a perfect gas.

3. The Sedov solution under the adjoint transformation. We now apply in a similar fashion the adjoint transformation given by Rogers [18] to the Sedov solution (2.1)-(2.5). Thus, if $p(x, t), \rho(x, t), u(x, t)$, given by (2.1)-(2.5), constitute a solution of equations (1.1)-(1.3), so also does the four-parameter adjoint system

$$
\begin{aligned}
& \bar{u}(\bar{x}, \bar{t})=a_{1} \rho u /\left(p+\rho u^{2}+a_{2}\right), \\
& \bar{\rho}(\bar{x}, \bar{t})=a_{3}\left(p+\rho u^{2}+a_{2}\right) / \rho\left(p+a_{2}\right), \\
& \bar{p}(\bar{x}, \bar{t})=a_{4}+\left(a_{1}{ }^{2} a_{3} /\left(p+\rho u^{2}+a_{2}\right)\right)
\end{aligned}
$$

in the $(\bar{x}, \bar{t})$-space where, as before, the $a_{i}(i=1,2,3,4)$ are arbitrary constants, and the adjoint space is defined by

$$
\begin{aligned}
d \bar{x} & =\rho d x-\rho u d t, \\
a_{1} d \bar{t} & =\rho u d x-\left(p+\rho u^{2}+a_{2}\right) d t .
\end{aligned}
$$

Along the particle trajectories we have $d \bar{x}=\bar{u} d \bar{t}$; in view of (3.1), (3.4) and (3.5) this reduces to

$$
a_{1} \rho\left(p+a_{2}\right) d x=0,
$$

or $x=$ constant $=b$, say $\left(a \rho \neq 0, p \neq-a_{2}\right)$. Upon setting

$$
\begin{gathered}
\frac{p_{0} \alpha^{(1+\epsilon) / 2 \epsilon} t_{0}}{\rho_{0}{ }^{\gamma-1}}=1, \quad \frac{(1-\epsilon)^{2} \rho_{0} x_{0}^{2} \alpha^{(1-\epsilon) / 2 \epsilon}}{t_{0}}=1, \\
a_{2} t_{0}=1, \quad \rho_{0} \alpha^{(1-\epsilon) / 2 \epsilon}(1-\epsilon) b=1, \\
x_{0}=1, \quad a_{1}=\epsilon\left(1-\epsilon^{2}\right)^{-1},
\end{gathered}
$$

the particle trajectories are given by 


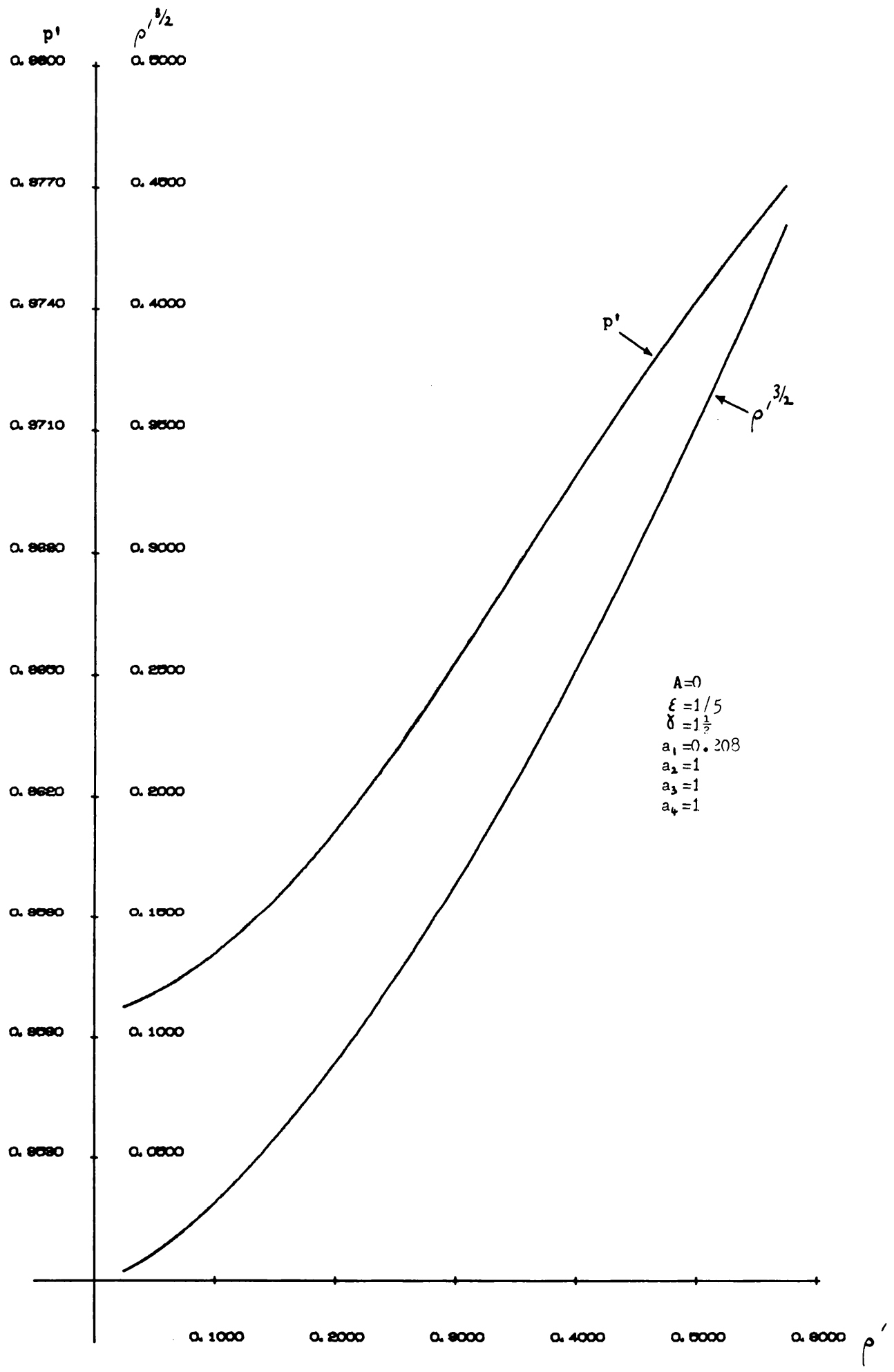

Fig. 2. Reciprocal transformation: $p^{\prime}$ and $\rho^{\prime} \gamma$ against $\rho^{\prime}$ on particle trajectory $A=0$. 


$$
\begin{aligned}
\bar{x} & =-\int\left[\left(1+b^{2} \sigma^{2 \epsilon-2}\right) \sigma^{-2 \epsilon}\right]^{(1-\epsilon) / 2 \epsilon} \sigma^{\epsilon-1}\left(1+\sigma^{-2 \epsilon}\right)^{1 / 2}\left(1+\sigma^{2 \epsilon}\right)^{-1 / 2} d \sigma \\
a_{1} \bar{t}= & -\int\left\{\left[\left(1+b^{2} \sigma^{2 \epsilon-2}\right) \sigma^{-2 \epsilon}\right]^{(1+\epsilon) / 2 \epsilon}\right. \\
& \left.\quad+\left[\left(1+b^{2} \sigma^{2 \epsilon-2}\right) \sigma^{-2 \epsilon}\right]^{(1-\epsilon) / 2 \epsilon} b^{2} \sigma^{2 \epsilon-2}\left(1+\sigma^{-2 \epsilon}\right)+1\right\}\left(1+\sigma^{2 \epsilon}\right)^{-1 / 2} d \sigma .
\end{aligned}
$$

It is readily verified that along these trajectories $(x=b)$

$$
\begin{aligned}
& p=\frac{1}{t_{0}}\left[\left(1+b^{2} \sigma^{2 \epsilon-2}\right) \sigma^{-2 \epsilon}\right]^{(1+\epsilon) / 2 \epsilon} \\
& \rho=\frac{t_{0}}{(1-\epsilon)^{2}}\left[\left(1+b^{2} \sigma^{2 \epsilon-2}\right) \sigma^{-2 \epsilon}\right]^{(1-\epsilon) / 2 \epsilon} \\
& u=\frac{(1-\epsilon)}{t_{0}} b \sigma^{\epsilon-1}\left(1+\sigma^{-2 \epsilon}\right)^{1 / 2}
\end{aligned}
$$

in general, and for $\epsilon=\frac{1}{2}$,

$$
\begin{aligned}
& p=\frac{1}{t_{0}}\left(b^{2}+\sigma\right)^{3 / 2} \sigma^{-3} \\
& \rho=4 t_{0}\left(b^{2}+\sigma\right)^{1 / 2} \sigma^{-1} \\
& u=\frac{b}{2 t_{0}}(1+\sigma)^{1 / 2} \sigma^{-1} .
\end{aligned}
$$

Substituting these values in (3.1)-(3.3) yields $\bar{p}$ and $\bar{\rho}$ as functions of $\sigma$ along the particle trajectories for $\epsilon=\frac{1}{2}$. The effect of this substitution is shown in Fig. $3 ; \bar{p}$ is plotted against $\bar{\rho}$ in the two cases $b=0, b=1$ with $\epsilon=\frac{1}{2}, a_{1}=0.667, a_{2}=a_{3}=a_{4}=1$ in each case. For comparison, $\bar{\rho}^{\gamma}=\bar{\rho}^{3}$ is plotted in addition and here it is seen that the agreement between shapes is rather less good than is the case for the reciprocal transformation.

4. Use of the adjoint transformation in studying piston-driven shocks. In a manner similar to that employed by Ustinov [13], we indicate an application of the adjoint transformation in examining the one-dimensional adiabatic motion of a gas in the region between a piston and a strong shock wave which arises as a result of the piston following an arbitrary law of motion and moving into a gas at rest. If $U_{0}(t)$ is the speed of the shock wave, $\rho_{0}(x)$ is the density distribution of the gas at rest into which it moves, then the conditions of conservation of mass and momentum through the shock may be written as

$$
\begin{aligned}
\rho_{0} U_{0} & =\rho\left(U_{0}-u\right), \\
p & =\rho_{0} u U_{0}, \\
d x & =U_{0} d t \\
p_{0} & \equiv 0
\end{aligned}
$$

where $p(x, t), \rho(x, t)$ and $u(x, t)$ is the solution for the gas flow between the piston and the shock. 


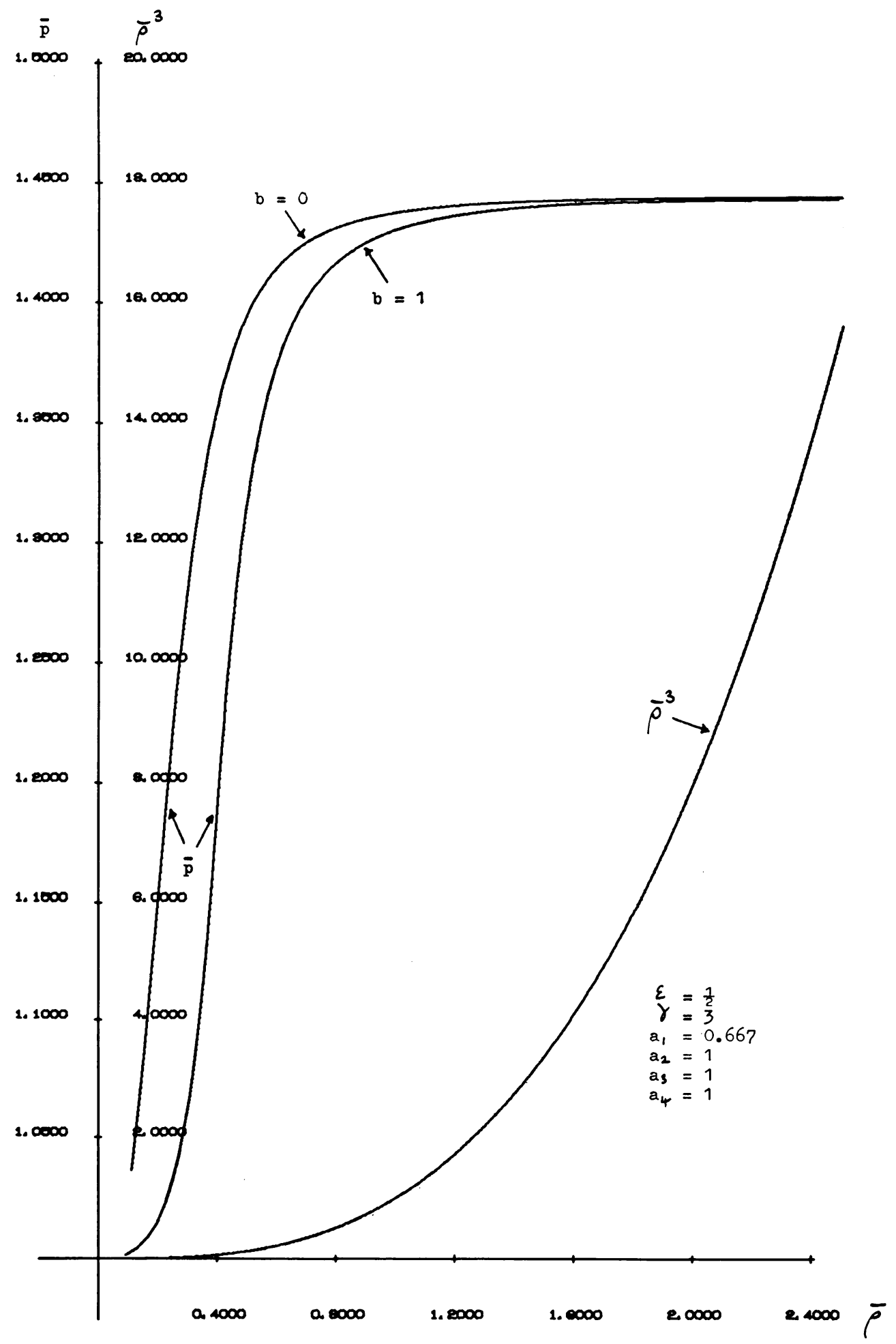

FIG. 3. Adjoint transformation: $\bar{p}$ and $\bar{\rho}^{\gamma}$ against $\bar{\rho}$ on particle trajectories $b=0,1$. 
Now suppose we have a solution set $\bar{p}(\bar{x}, \bar{t}), \bar{\rho}(\bar{x}, \bar{t}), \bar{u}(\bar{x}, \bar{t})$ for homentropic flow in the adjoint space. Then a new solution set $p(x, t), \rho(x, t), u(x, t)$ is given by the inverse transformation to be (Rogers [18])

$$
\begin{aligned}
& p(x, t)=-a_{2}+\frac{a_{1}{ }^{2} a_{3}}{\left(\bar{p}+\bar{\rho} \bar{u}^{2}-a_{4}\right)}, \\
& \rho(x, t)=\frac{a_{3}\left(\bar{p}+\bar{\rho} \bar{u}^{2}-a_{4}\right)}{\bar{\rho}\left(\bar{p}-a_{4}\right)}, \\
& u(x, t)=\frac{a_{1} \bar{\rho} \bar{u}}{\left(\bar{p}+\bar{\rho} \bar{u}^{2}-a_{4}\right)},
\end{aligned}
$$

where

$$
\begin{gathered}
\rho d x-\rho u d t=d \bar{x} \\
\rho u d x-\left(p+\rho u^{2}+a_{2}\right) d t=\dot{a}_{1} d \bar{t}
\end{gathered}
$$

Since (4.8) and (4.9) may be rewritten as

$$
\begin{aligned}
& d x=\frac{\left(p+\rho u^{2}+a_{2}\right)}{\rho\left(p+a_{2}\right)} d \bar{x}-\frac{u a_{1}}{\rho\left(p+a_{2}\right)} d \bar{t}, \\
& d t=\frac{u}{\left(p+a_{2}\right)} d \bar{x}-\frac{a_{1}}{\left(p+a_{2}\right)} d \bar{t},
\end{aligned}
$$

then $p, \rho, u$ may be derived as functions of $x$ and $t$ by combining (4.5)-(4.7) with (4.10) and (4.11).

Now it may readily be shown that, with $a_{2}=0$, along $d x / d t=U_{0}, \bar{t}$ is constant, so that evidently the law of motion of the piston is given by $\bar{x}=0, \bar{t} \leq 0$, while that of the shock is given by $\bar{t}=0, \bar{x} \geq 0$. That is, to each homentropic solution $\bar{p}(\bar{x}, \bar{t}), \bar{\rho}(\bar{x}, \bar{t})$, $\bar{u}(\bar{x}, \bar{t})$ of the one-dimensional non-steady gasdynamic equations there corresponds a three-parameter family of solutions, (4.5)-(4.7) with $a_{2}=0$, and, further, this family of solutions is that of a flow of a gas between a piston and a non-uniform shock moving into a gas at rest provided the law of motion of the piston and of the shock are given by

$$
\begin{gathered}
d x=u d t, \quad \bar{t} \leq 0, \\
U_{0}(t)=d x / d t=\left(p+\rho u^{2}\right) / \rho u, \quad \bar{x} \geq 0
\end{gathered}
$$

respectively, where the relation between the $(x, t)$ and $(\bar{x}, \bar{t})$ spaces is given by $(4.8)$ and (4.9). In addition to the conservation of mass and momentum conditions there will also be a conservation of energy requirement to be met which will limit solutions found.

As an example, consider a family of solutions given by Ustinov [13] for high-temperature flow of a perfect gas, when the temperature may be considered to depend only on the time. In this case

$$
\begin{gathered}
\bar{\rho}=V^{\gamma /(1-\gamma)}, \quad \bar{p}=(\gamma-1) f_{1}(\bar{t}) V^{\gamma /(1-\gamma)} \\
\bar{u}=\phi(\bar{t})(\bar{x}+h), \quad V=\psi(\bar{t})(\bar{x}+h)^{2} \\
\phi(\bar{t})=-\frac{\gamma}{(\gamma+1)} \frac{\psi^{\prime}}{\psi}, \quad f_{1}(\bar{t})=\frac{\phi^{\prime}+\phi^{2}}{2 \psi}
\end{gathered}
$$


where $h$ is a constant. The family may be considered parameterized in terms of the arbitrary function of time $\psi(\bar{t})$. If this solution is now to be used to generate a pistondriven shock flow of a perfect gas in the $(x, t)$-space, then $\psi(\bar{t})$ and the available parameters must be so chosen that the equation of state

$$
p=\text { constant } \cdot \rho^{n}
$$

and the energy equation

$$
\frac{1}{2} U_{0}^{2}=\frac{n}{(n-1)} \frac{p}{\rho}+\frac{1}{2} u^{2}
$$

are satisfied for $\bar{t}=0, \bar{x} \geq 0$ when $p, \rho, u$ and $U_{0}$ are evaluated from (4.5)-(4.7) and (4.13).

Acknowledgements. Figs. 1-3 were produced by the digital graph-plotter of the British Aluminium Company's (Research Division) ICL 4120 computer. The use of time on this machine is gratefully acknowledged.

\section{REFERENCES}

[1] H. Bateman, Proc. Nat. Acad. Sci. U.S.A. 24, 246-251 (1938)

[2] A. Haar, Math. Ann. 100, 481-502 (1928)

[3] R. C. Prim, J. Appl. Phys. 20, 448-450 (1949)

[4] H. S. Tsien, Two-dimensional subsonic flow of compressible fluids, J. Aero. Sci. 6, 399-407 (1939)

[5] H. Bateman, Quart. Appl. Math. 1, 281-298 (1944)

[6] G. Power and P. Smith, Applications of a reciprocal property to subsonic flow, Appl. Sci. Res. A8, 386-392 (1959); Reciprocal properties of plane gas flows, J. Math. Mech. 10, 349-361 (1961)

[7] G. Power and A. Tunbridge, Reciprocal properties in magnetogasdynamics, ZAMM 43, 191-197 (1963)

[8] G. Power and D. Walker, Some reciprocal relations in rotational magnetogasdynamic flow, ZAMP $15,2,144-154$ (1964)

[9] A. A. Nikol'skii, Invariant transformations of the equations of motion of an ideal monatomic gas and new classes of their exact solution, P.M.M. 27, 740-756 (1963)

[10] P. Smith, An extension of the substitution principle to certain unsteady gas flows, Arch. Rat. Mech Anal. 15, 147-153 (1964)

[11] E. D. Tomilov, On the method of invariant transformations of the gas-dynamic equations, P.M.M. 29, 959-960 (1965)

[12] V. A. Rykov, On an exact solution of the equations of magnetogasdynamics of finite conductivity, P. M. M. 29, 178-181 (1965)

[13] M. D. Ustinov, Transformation and some solutions of the equations of motion of an ideal gas, Izv. AN SSSR Mekh. Zhid. I Gaza (Fluid Dynamics) 3, 68-74 (1966); Ideal gas flow behind a finiteamplitude shock wave, Izv. AN SSSR Mekh. Zhid. I Gaza 2, 1, 88-90 (1967)

[14] L. A. Movsesian, On an invariant transformation of equations of one-dimensional unsteady motion of an ideal compressible fluid, P.M.M. 31, 137-141 (1967)

[15] M. H. Martin, A new approach to problems in two-dimensional flow, Quart. Appl. Math. 8, 137-150 (1950); The propagation of a plane shock into a quiet atmosphere, Canadian J. Math. 5, 37-39 (1953)

[16] L. I. Sedov, On the integration of the equations of one-dimensional motion of a gas, DAN SSSR 90, $5(1953)$

[17] C. Rogers, Reciprocal relations in non-steady one-dimensional gasdynamics, ZAMP 19, 1, 58-63 (1968)

[18] C. Rogers, Invariant transformations in non-steady gasdynamics and magnetogasdynamics, ZAMP 20, 3, 370-382 (1969) 